\section{Characterising the immune profile of the kidney biopsy at lupus nephritis flare differentiates early treatment responders from non-responders}

\author{
Samir V Parikh, ${ }^{1}$ Ana Malvar, ${ }^{2}$ Huijuan Song, ${ }^{1}$ Valeria Alberton, ${ }^{3}$ Bruno Lococo, ${ }^{2}$ \\ Jay Vance, ${ }^{1}$ Jianying Zhang, ${ }^{4}$ Lianbo $\mathrm{Yu},{ }^{2}$ Brad $\mathrm{H}$ Rovin ${ }^{1}$
}

To cite: Parikh SV, Malvar A, Song $\mathrm{H}$, et al. Characterising the immune profile of the kidney biopsy at lupus nephritis flare differentiates early treatment responders from non-responders. Lupus Science \& Medicine 2015;2: e000112. doi:10.1136/lupus2015-000112

- Additional material is available. To view please visit the journal (http://dx.doi.org/ 10.1136/lupus-2015000112).

Received 24 June 2015 Revised 12 August 2015 Accepted 2 September 2015

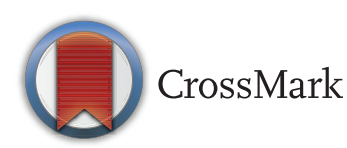

${ }^{1}$ Division of Nephrology, The Ohio State University Wexner Medical Center, Columbus, Ohio, USA

${ }^{2}$ Nephrology Unit, Hospital Fernandez, Buenos Aires, Argentina

${ }^{3}$ Department of Pathology, Hospital Fernandez, Buenos Aires, Argentina

${ }^{4}$ Center for Biostatistics, The Ohio State University Wexner Medical Center, Columbus, Ohio, USA

Correspondence to Dr Brad H Rovin; rovin.1@osu.edu

\section{ABSTRACT}

Introduction: The kidney biopsy is used to diagnose and guide initial therapy in patients with lupus nephritis (LN). Kidney histology does not correlate well with clinical measurements of kidney injury or predict how patients will respond to standard-of-care immunosuppression. We postulated that the gene expression profile of kidney tissue at the time of biopsy may differentiate patients who will from those who will not respond to treatment.

Methods: The expression of 511 immune-response genes was measured in kidney biopsies from 19 patients with proliferative LN and 4 normal controls. RNA was extracted from formalin-fixed, paraffinembedded kidney biopsies done at flare. After induction therapy, 5 patients achieved a complete clinical response (CR), 10 had a partial response (PR) and 4 patients were non-responders (NRs). Transcript expression was compared with normal controls and between renal response groups.

Results: A principal component analysis showed that intrarenal transcript expression from normal kidney, CR biopsies and NR biopsies segregated from each other. The top genes responsible for CR clustering included several interferon pathway genes (STAT1, IRF1, IRF7, MX1, STAT2, JAK2), while complement genes (C1R, $C 1 Q B, C 6, C 9, C 5, M A S P 2)$ were mainly responsible for NR clustering. Overall, 35 genes were uniquely expressed in NR compared with CR. Pathway analysis revealed that interferon signalling and complement activation pathways were upregulated in both groups, while BAFF, APRIL, nuclear factor- $\mathrm{B}$ B and interleukin-6 signalling were increased in CR but suppressed in NR.

Conclusions: These data suggest that molecular profiling of the kidney biopsy at LN flare may be useful in predicting treatment response to induction therapy.

\section{INTRODUCTION}

The percutaneous kidney biopsy is the gold standard for the diagnosis of glomerular diseases. Perhaps more so than for any other glomerular disease, biopsy findings are used

\section{KEY MESSAGES}

- Not all LN is created equal: The molecular profile of LN flares that end in complete response differs from flares that end in no response.

- Molecular profiling of the kidney biopsy at LN flare may help guide treatment and better predict response.

- The addition of molecular analysis to routine histology will help facilitate the personalization of LN treatment.

to classify and subgroup lupus nephritis (LN) in order to better inform treatment decisions and predict prognosis. ${ }^{1} 2$ Several schemas have been used to classify LN biopsies, the most recent being the 2004 International Society of Nephrology (ISN) and Renal Pathology Society (RPS) classification. ${ }^{2} \quad 3$ Although the objective of the ISN/RPS classification was to align histology with outcomes, ${ }^{3-5}$ little progress has been made in using the kidney biopsy to predict treatment response in LN. Contributing to this is the poor correlation between clinical findings and renal histology. $^{6-9}$ A possible explanation for the discordance between clinical and histological findings is that the histological responses of the kidney to injury are limited, whereas the pathogenic mechanisms of renal injury in LN are diverse. It is likely that molecular analysis of kidney biopsies will provide more information about how the kidney will respond to treatment than histology alone.

To test this hypothesis, we measured transcript expression of a panel of immune response genes in the diagnostic kidney biopsies of patients with proliferative LN and evaluated their expression profile at flare. Gene expression profiles were compared between those who achieved a complete clinical response and those who did not achieve a 
response after standard-of-care LN induction therapy. Differentially expressed genes were subject to informatics analyses to predict the immune signalling pathways that differentiated responders and non-responders (NRs) at the start of treatment.

\section{METHODS}

Kidney biopsies

For this proof-of-concept study, transcript expression was measured in the kidney biopsies of 19 patients with proliferative (class III or IV \pm V) LN. These biopsies were done from 2007 to 2011. The biopsies had been archived after all clinical testing was completed. The use of these biopsies was approved by the Hospital Fernandez ethics board.

As a control, archived kidney tissue from preimplantation biopsies of living-donor kidneys $(n=4)$ was analysed in parallel with the LN biopsies. Pre-implantation biopsies are done on all donor kidneys at the Ohio State University Wexner Medical Center as part of the clinical transplant protocol. The use of these biopsies was approved by The Ohio State University Institutional Review Board.

\section{Treatment protocols and outcomes}

All patients were treated with standard-of-care immunosuppression protocols. Sixty-three per cent of the cohort received $2000-3000 \mathrm{mg} /$ day mycophenolate mofitel, while $37 \%$ were given $750-1000 \mathrm{mg} /$ month of intravenous cyclophosphamide. All patients received a corticosteroid taper starting with $1 \mathrm{mg} / \mathrm{kg} /$ day prednisone at the beginning of induction. The induction period lasted, in general, 6 months.

The serum creatinine (SCr) concentration and $24 \mathrm{~h}$ urine protein level were available on all patients at flare and after finishing induction treatment. Complete renal response was defined as having an improvement in proteinuria to $<0.5 \mathrm{~g} /$ day with normalisation of SCr. Partial renal response (PR) was defined as at least a $50 \%$ reduction in proteinuria, to a level $<3 \mathrm{~g} /$ day, but $>0.5 \mathrm{~g} /$ day, with stable or improved SCr. ${ }^{10}$ Patients who did not meet either of these criteria were defined as NRs.

\section{RNA extraction and analysis}

All biopsies used in this study had been formalin-fixed and paraffin-embedded (FFPE). Ten micron sections were cut from the paraffin blocks, and for each biopsy two sections were deparaffinised and digested with proteinase K. DNA was removed with DNase. RNA was precipitated and the precipitate was added to RNeasy MinElute spin columns (Qiagen, Redwood City, California, USA). RNA was eluted in RNase-free water. The complete details of FFPE deparaffinisation and RNA extraction can be found in online supplementary methods 1.

Gene transcript expression was analysed from $250 \mathrm{ng}$ of extracted RNA using the Nanostring ncounter platform and the GX human immunology transcript panel (Nanostring Technologies, Seattle, Washington, USA) ${ }^{11-13}$ The ncounter platform was chosen because it is superior to microarray for quantification of gene expression in FFPE samples. ${ }^{14}{ }^{15}$ The human immunology panel consisted of 511 immune response genes, 6 positive control genes and 6 negative control genes. A complete list of these genes can be found in online supplementary table S1.

Multiplex RT-PCR using TaqMan Gene expression assays (Applied Biosystems/Life Technology, Grand Island, New York, USA, catalogue \# 4384267) was done to verify Nanostring results for a subset of differentially expressed transcripts. Using a high-capacity RNA-to-cDNA kit (Applied Biosystems/Life Technology, catalogue \# 4387406), cDNA was generated from $300 \mathrm{ng}$ total tissue RNA and data were collected during RT-PCR cycles using gene-specific fluorogenic probes. The complete details of the RT-PCR protocol can be found in online supplementary methods 2 .

\section{Statistical analysis}

Before statistical analyses, raw gene expression data were normalised to the positive spike-in controls and then $\log 2$ transformed. To reduce the false positive rate, only genes with an expression level at least 2 SDs above the mean expression of the negative controls were included in the analysis. Quantile normalisation was used for normalisation across samples. Overall, 382 transcripts survived normalisation and were further analysed for differential expression.

Patients were stratified by response status (CR, PR and NR). Transcript expression levels were compared with normal kidney tissue and between responder groups to identify gene signatures and/or pathways that differentiated responders from NRs.

Descriptive statistics are presented as mean $\pm \mathrm{SD}$ or as a percentage. For clinical variables, $t$ tests, analysis of variance model or Wilcoxon rank-sum tests were applied as appropriate, followed by Bonferroni correction for multiple comparisons. For categorical clinical variables, Fisher's exact test was used. Adjusted p values were reported for each comparison and were considered significant if $<0.05$.

A linear model was used to compare the gene expression of normal kidney tissue to $\mathrm{LN}$ biopsies from patients who achieved a CR, PR or NR after induction therapy, and also to directly compare gene expression between the three LN responder groups. In order to improve the estimates of variability and differential expression, variance smoothing methods were employed. ${ }^{16} \mathrm{p}$ Values were adjusted by controlling the mean number of false positives at 4 out of 400 genes (ie, $\alpha=0.01$ ). To be considered differentially expressed, at least a twofold difference in transcript levels and a $p$ value $<0.01$ were required for any specific gene.

The RNA analysis for this cohort was conducted in two batches with 9 samples in the first batch and 10 
samples in the second batch. The control samples were the same for both batches. To prevent confounding from batch effect, a batch effect adjustment was applied for testing expression differences in the linear model and to standardise the data across the batches.

\section{Pathway analysis}

Ingenuity Pathway Analysis (IPA, Qiagen) was used to identify canonical pathways that were differentially expressed between the treatment response groups and controls, and between each treatment response group. For each comparison, all 382 genes were analysed for significance. For IPA analysis, transcripts with a $p$ value $<0.05$ and at least a 1.5 -fold change were included in the analysis. These criteria were used to include as many significant differentially expressed and biologically relevant genes as possible to enrich pathway analyses. Comparing CR to control, 68 transcripts met the criteria and were included in the analysis. Comparing NR to control, 138 transcripts met the criteria and were included in the analysis. The Ingenuity Knowledge Base was used as the reference set against which the significant transcripts were compared for enrichment. Significance of upregulated or downregulated pathways was determined using Fisher's exact test and is presented as the negative logarithm of the $p$ value ( $-\log$ ( $p$ value $))$. A multiple corrections test is not available for IPA; therefore, all values are reported as unadjusted $\mathrm{p}$ values. The predicted activation state (upregulated or downregulated) of significantly expressed pathways was determined by a z-score algorithm that compared the gene expression data set with the expected canonical pathway patterns (http:// ingenuity.force.com/ipa).

\section{RESULTS}

\section{Characteristics of the LN cohort}

The demographic, clinical and pathological characteristics of the LN patients segregated by renal response after initial therapy are given in table 1 . The median time to follow-up was 10 months (range 6-37 months). All patients were Caucasian and Hispanic. Although the clinical and histological findings at biopsy were generally similar between response groups, NR had more proteinuria at flare. Additionally, $40 \%$ of the flares in the CR group were relapses, while in NR $75 \%$ were relapses $(\mathrm{p}=\mathrm{NS})$. For relapsing patients, their prior flares occurred two or more years prior to the current flare event. Immunosuppressive treatment prior to the kidney biopsy was similar between the groups. Two patients in the CR group were on azathioprine prior to the kidney biopsy while three patients were on prednisone only ( $\leq 10 \mathrm{mg} /$ day). In the NR group, one patient was not on any immunosuppressive therapy prior to biopsy, two were on prednisone only ( $\leq 10 \mathrm{mg} /$ day) and one patient was on azathioprine. For the PR group, four patients were off immunosuppression at the time of kidney biopsy, four patients were on prednisone only ( $\leq 20 \mathrm{mg}$ /

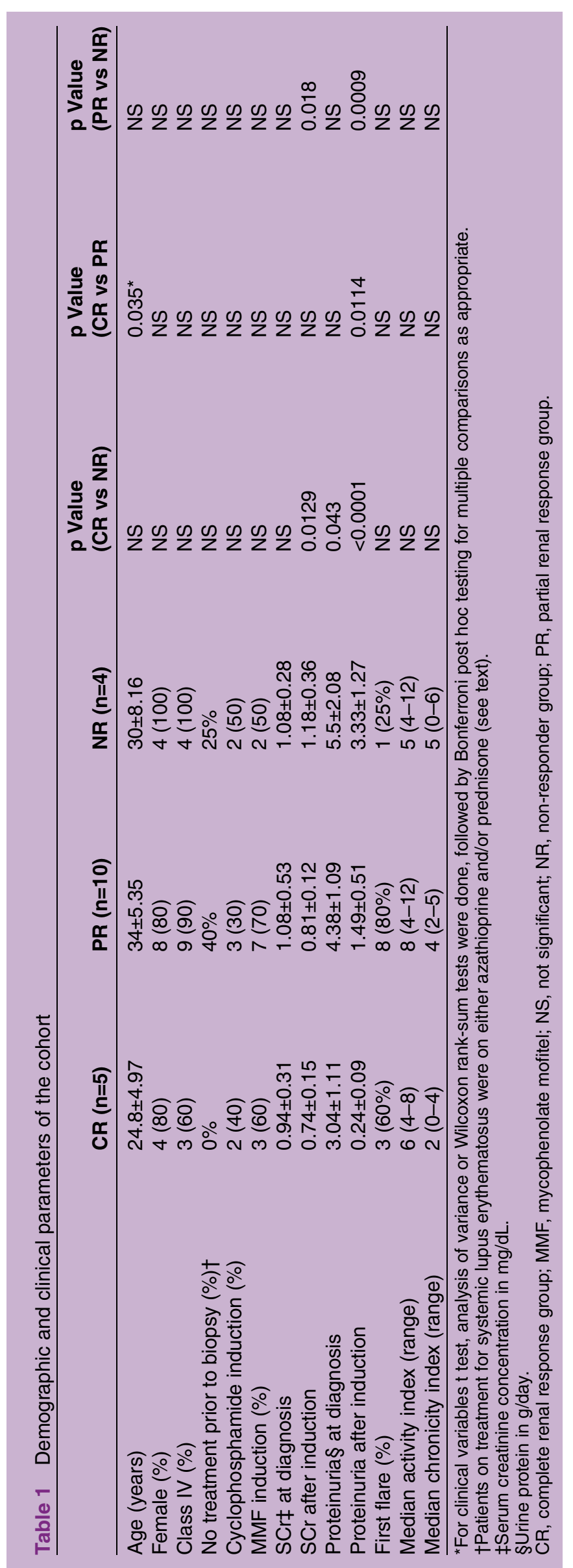


A

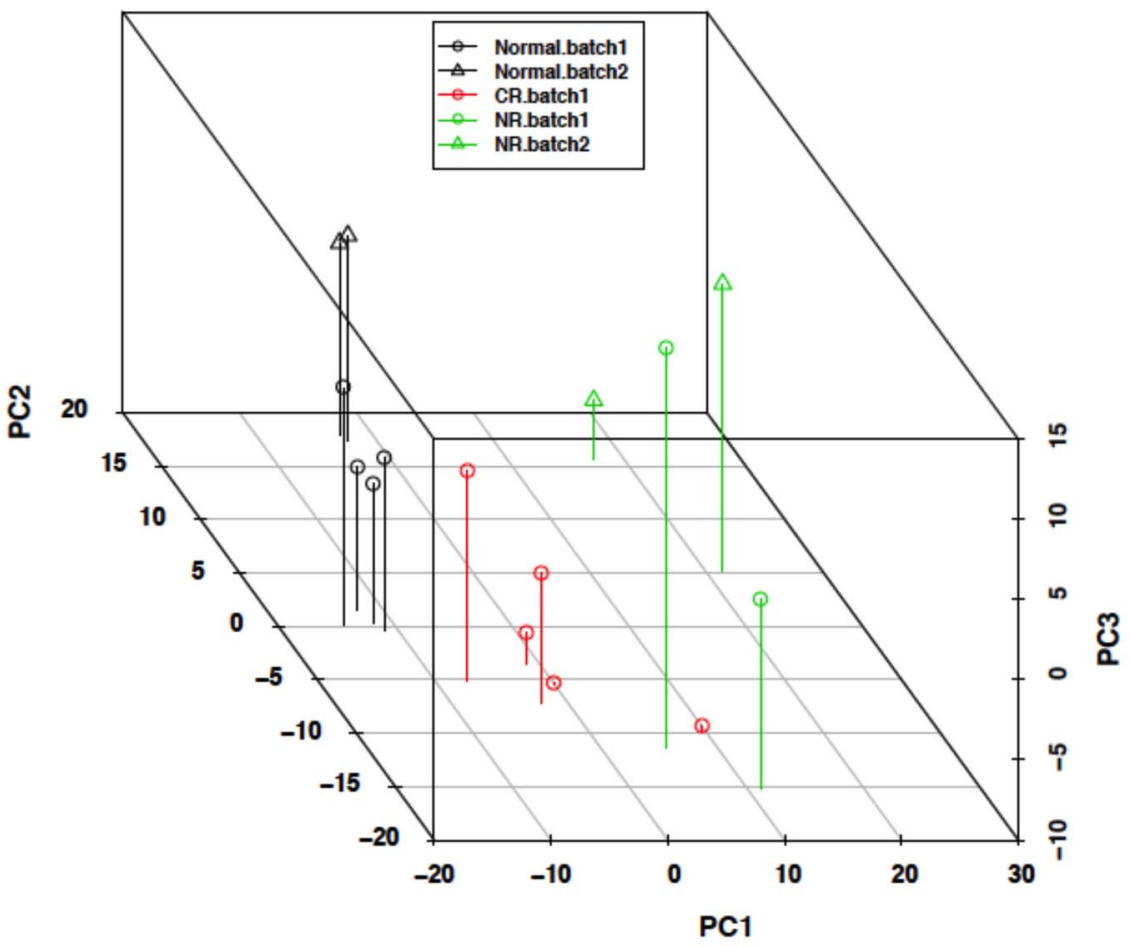

B
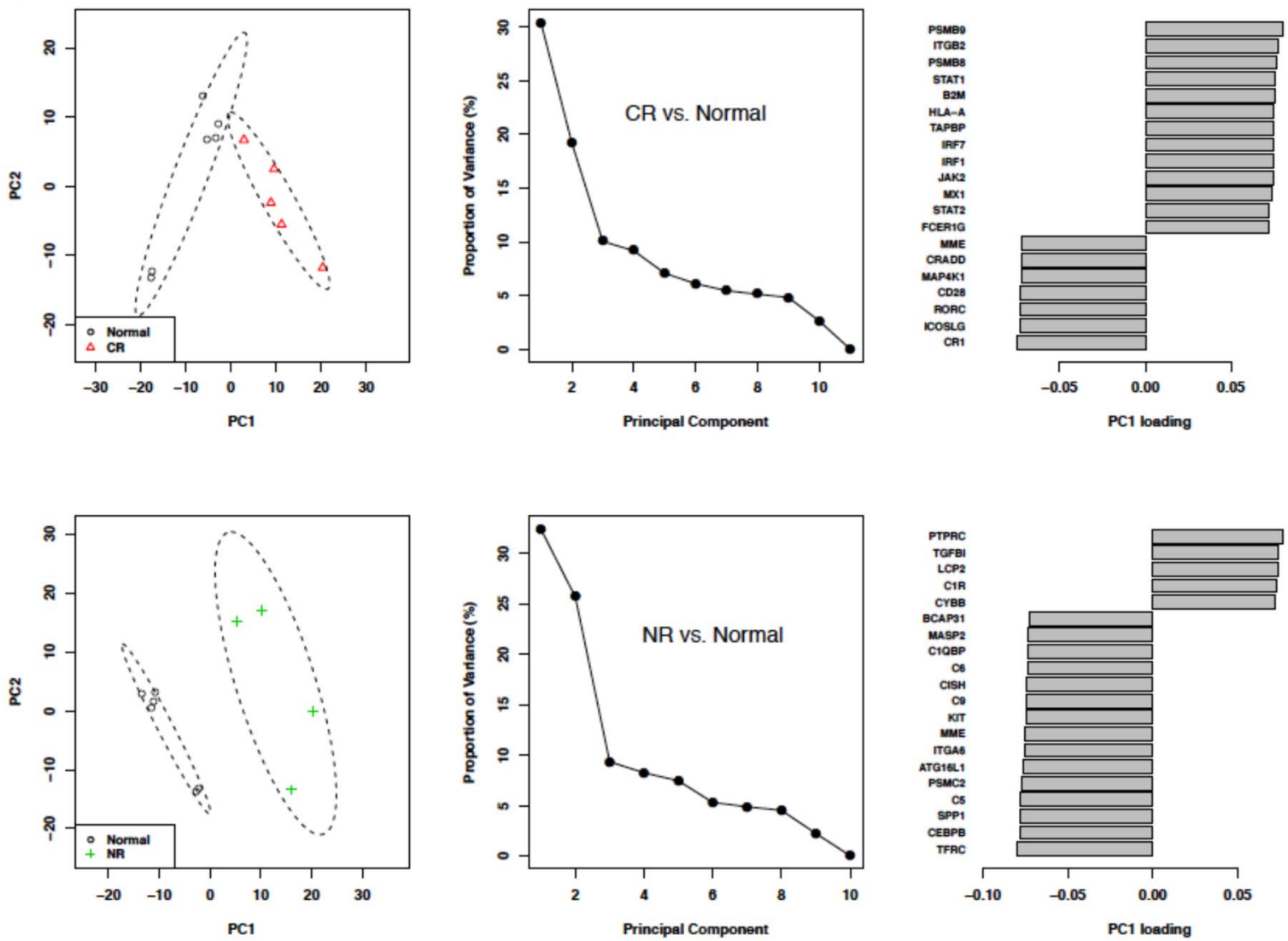

Figure 1 Principal component analysis (PCA) of immune response gene expression in kidney biopsies. (A) The PCA based on gene expression data for complete responders (CRs), non-responders (NRs) and normal controls. The RNA analysis of this biopsy set was conducted in two batches. A batch effect adjustment was applied to prevent confounding and both batches are represented in the figure (batch 1, circles; batch 2, triangles). The normal controls were common to both batches. The PCA shows that CR (except for one patient), NR and normal controls groups clustered separately from each other. (B) A factor loading plot using flare data from CR and NR to identify the genes important for the clustering seen in the PCA. Principal component 1 (PC1) was used as it accounted for the highest proportion (35-50\%) of variance. For each PC1 loading plot, only the top 20 genes ranked by absolute factor loadings for PC1 were selected. The plots show the top genes contributing to group clustering. 
day) and two patients were on azathioprine. Activity and chronicity indices were not significantly different between the responder groups. After induction treatment, $\mathrm{SCr}$ and proteinuria improved in the CR and PR groups, but $\mathrm{SCr}$ worsened in the NR group.

A principal component analysis (PCA) of immune gene transcripts from normal kidney and the LN responder groups was done (figure 1A). This showed that normal controls clustered together and were separated from the LN responder groups. Additionally, all but one CR patient clustered together and separately from NR. The individual patients of the PR group did not cluster, but instead were distributed between the CR and NR clusters (data not shown).

Factor-loading plots for each principal component were created to identify the genes contributing to group clustering. ${ }^{17}$ Because principal component 1 (PC1) seemed to best associate with group clustering, the top 20 genes for PC1 were determined after batch adjustment (figure 1B). The interferon-inducible genes STAT1, IRF1, IRF7, MX1, STAT2 and JAK2 contributed prominently to CR clustering. In addition, the proteasome genes PSMB8 and PSMB9, known to be induced by gamma interferon, ${ }^{18}$ and the $\mathrm{T}$ cell co-stimulation genes CD28 and ICOSLG were important for CR clustering.

Contributing significantly to NR clustering were genes for the complement components C1QBP, C1R, MASP, C6, C9 and C5. Additionally, TGFBI, CEBPB and SPP1 were also important for NR clustering (figure 1B). $T G F B I$ is known to promote renal fibrosis and has previously been implicated in $\mathrm{LN}^{19-21} C E B P B$ encodes the CCAAT/enhancer binding protein- $\beta$ and is necessary for macrophage-mediated removal of apoptotic debris. ${ }^{22}$ Osteopontin (SPP1) is produced by various immune cells and is important for regulating several aspects of the immune system including T-helper cell balance and B cell production of antibodies. ${ }^{23}$ Overexpression of osteopontin has been implicated in the development of murine $\mathrm{LN}^{24}$ and is associated with human systemic lupus erythematosus (SLE). ${ }^{25}$

\section{Immune gene expression profiles in kidney biopsies at LN flare}

Overall, 71 transcripts were differentially expressed in LN kidneys compared with normal control kidneys (figure 2). Of these, 19 transcripts were common to each LN response type (figure 2). Transcripts with altered expression in $\mathrm{LN}$ that were unique to each response group are listed in table 2.

CR renal tissue showed three unique transcripts, including human leukocyte antigen (HLA-A) and interferon-induced helicase $C$ domain protein 1 (IFIH1), which were upregulated relative to control, and nuclear factor of activated T cells, cytoplasmic 1 (NFATC1), which was downregulated. IFIH1 is a cytoplasmic dsRNA sensor important for activating interferon-alpha, and has been shown to promote apoptosis, inflammation and

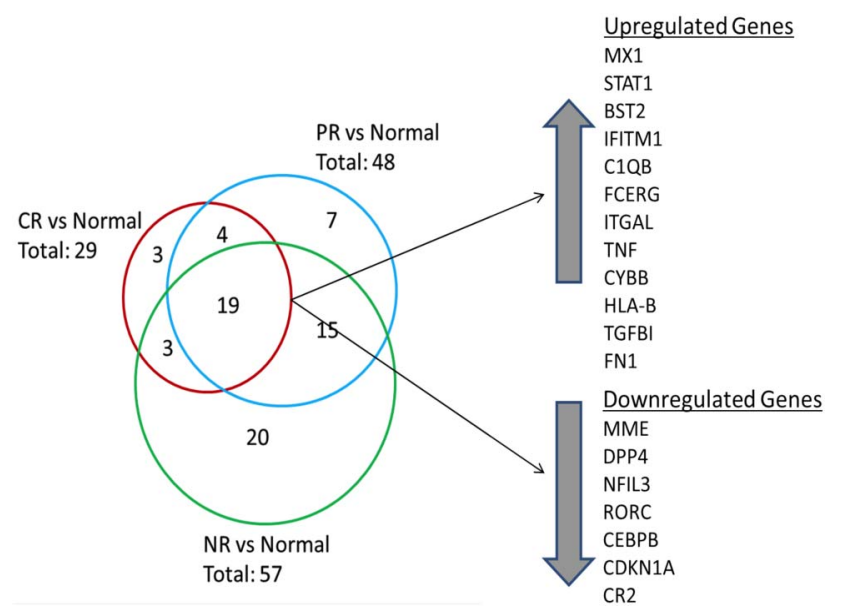

Figure 2 Differential renal gene expression at flare for each lupus nephritis (LN) responder group compared with normal kidney. The Venn diagram shows the number of common and unique genes in each LN responder group. The 19 genes that were differentially expressed between all LN groups and normal tissue are listed. Twelve genes were upregulated and seven genes were downregulated. Three genes were uniquely expressed in the CR group compared with normal while seven genes were uniquely expressed in the partial response (PR) group. A 20-gene signature differentiated non-responders (NR) from CR and PR groups.

autoantibody production in SLE. ${ }^{26}$ NFATC1 regulates T cell proliferation and differentiation and can be blocked by calcineurin inhibitors. ${ }^{27}$

Seven transcripts were unique to PR, including increased expression of complement factor $B(C F B)$, and decreased expression of interleukin-1 receptor-like-1 (IL1RL1) and FK506 binding protein (FKBP5) (table 2). IL1RL1 is an IL-1 family member that binds IL-33 and regulates nuclear factor (NF)-kB-mediated Th2 immune responses. ${ }^{28}$ FKBP5 is an immunophilin family member that binds tacrolimus and rapamycin and is important for immune regulation.

Twenty transcripts were unique to NR, of which 12 were decreased relative to control and 8 were increased (table 2). Transcripts with decreased expression included fas-associated protein with death domain (FADD), a regulator of apoptosis, programmed-death ligand 1 (CD274/ $P D-L 1)$, which regulates autoreactive $\mathrm{T}$ cell production, ${ }^{29}$ interleukin-6 signal transducer (IL-6ST) and IL-6 receptor $(I L-6 R)$. Transcripts with increased expression in NR kidneys included IL-1 receptor antagonist (ILIRN), a natural antagonist of IL-1, chemokine $C$-X3-C motif receptor 1 (CXCR1), the receptor for a chemokine involved in migration and adhesion of leucocytes, and $T$ cell activation GTPase activating protein (TAGAP), which plays a role in T cell activation. ${ }^{30}$

When CR and NR were directly compared, five transcripts were found to be differentially expressed between these two extremes of clinical response. Membrane metal loendopeptidase $(M M E) \quad(\mathrm{p}=0.0034)$, a glycoprotein abundant in the proximal tubule of the kidney, $F A D D$ 
Table 2 Differentially expressed transcripts at flare in each lupus nephritis (LN) response group compared with normal controls

\begin{tabular}{|c|c|c|c|c|c|c|c|c|}
\hline \multicolumn{3}{|c|}{ Complete response group } & \multicolumn{3}{|c|}{ No response group } & \multicolumn{3}{|c|}{ Partial response group } \\
\hline Gene & Fold change* & p Valuet & Gene & Fold change* & p Valuet & Gene & Fold change $^{*}$ & p Valuet \\
\hline HLA-A & 2.17 & 0.0007 & LILRA3 & 2.23 & 0.0006 & $C F B$ & 2.2 & 0 \\
\hline IFIH1 & 2.16 & 0.0003 & CSF2RB & 2.23 & 0 & IL18R1 & 0.46 & 0 \\
\hline \multirow[t]{18}{*}{ NFATC1 } & 0.48 & 0.0002 & TAGAP & 2.21 & 0.0004 & KIR2DL5A & 0.46 & 0.0108 \\
\hline & & & BTK & 2.17 & 0.0004 & $N F K B I A$ & 0.44 & 0 \\
\hline & & & IL1RN & 2.11 & 0.0099 & $T A L 1$ & 0.41 & 0 \\
\hline & & & CX3CR1 & 2.07 & 0.0022 & IL1RL1 & 0.41 & 0.0011 \\
\hline & & & $\angle C P 2$ & 2.01 & 0.0001 & FKBP5 & 0.11 & 0 \\
\hline & & & FCGR2C & 2.01 & 0.0005 & & & \\
\hline & & & IGF2R & 0.49 & 0 & & & \\
\hline & & & NOS2 & 0.49 & 0.0013 & & & \\
\hline & & & $R E L B$ & 0.49 & 0.0118 & & & \\
\hline & & & $F A D D$ & 0.47 & 0 & & & \\
\hline & & & PLAU4 & 0.47 & 0.0029 & & & \\
\hline & & & ITGA6 & 0.46 & 0 & & & \\
\hline & & & $C D 274$ & 0.46 & 0.0001 & & & \\
\hline & & & IL6R & 0.46 & 0.0001 & & & \\
\hline & & & IL6ST & 0.46 & 0.0029 & & & \\
\hline & & & ICOSLG & 0.45 & 0 & & & \\
\hline & & & $B S T 1$ & 0.44 & 0.0003 & & & \\
\hline & & & $C D 81$ & 0.41 & 0 & & & \\
\hline
\end{tabular}

$(\mathrm{p}<0.0001)$ and CD274/PD-L1 $(\mathrm{p}=0.0002)$ were $\geq 2$-fold higher in CR. Complement component C1S ( $\mathrm{p}=0.003)$ and integrin beta-2 (ITGB2) $\quad(\mathrm{p}=0.0007)$ were $\geq 2$-fold lower in CR versus NR.

\section{RT-PCR measurement of selected transcripts}

MME, ITGB2, MX1, STAT1 and CCL19 mRNA levels were measured by RT-PCR and compared with the results obtained using the ncounter platform on the same biopsies to confirm trends identified by Nanostring. The selected transcripts were found to be differentially expressed between LN groups and controls by Nanostring, and the same trend in transcript expression was observed using RT-PCR (see online supplementary table S2).

\section{Differentially regulated pathways at flare}

To integrate all of the differentially expressed tissue transcripts into immunological pathways that may be relevant to kidney injury in LN, the expression differences of the genes listed in table 2 were analysed by IPA and differentially activated canonical signalling pathways were identified. Figure 3 shows the top differentially activated or suppressed pathways in CR and NR compared with control. Only immune pathways where an activation status could be predicted were reported. Interferon signalling, complement and leucocyte extravasation pathways were predicted to be activated in CR and NR, while PI3K signalling in B-lymphocytes was predicted to be suppressed (figure 3). Pathway analysis also identified several differences between CR and NR. For example, in
CR kidneys IL-6, NF-אB, B cell activating factor and tolllike receptor (TLR) signalling were predicted to be activated, whereas in NR kidneys these same pathways were predicted to be suppressed (figure 3).

\section{DISCUSSION}

The present work shows that the intrarenal transcript expression profile of the diagnostic kidney biopsy in LN differs between patients who had a rapid clinical response to induction therapy and patients who did not. This is the first study to examine the association of future clinical renal response with the molecular profile of the human LN kidney.

A subset of 19 immune response genes from the transcript panel used in this study was differentially expressed in all LN biopsies compared with normal kidney tissue. The protein products of these genes are consistent with our current understanding of how the immune system is dysregulated in SLE. For example, the expression of interferon and complement genes was significantly increased in LN kidneys, and both pathways appear to be involved in the pathogenesis of SLE and LN. ${ }^{31-38}$ Conversely, the expression of CEBPB was decreased in LN kidneys. Because macrophages lacking $C E B P B$ do not remove apoptotic debris very well, such debris may accumulate and contribute to kidney-specific autoimmunity and autoantibody production. ${ }^{22}$ 39-41

PCA of transcript expression revealed clustering of LN flare groups compared with controls. Factor loading plots of the PCA identified genes that were responsible for the 
A Top Activated/Suppressed immune pathways in CR flares

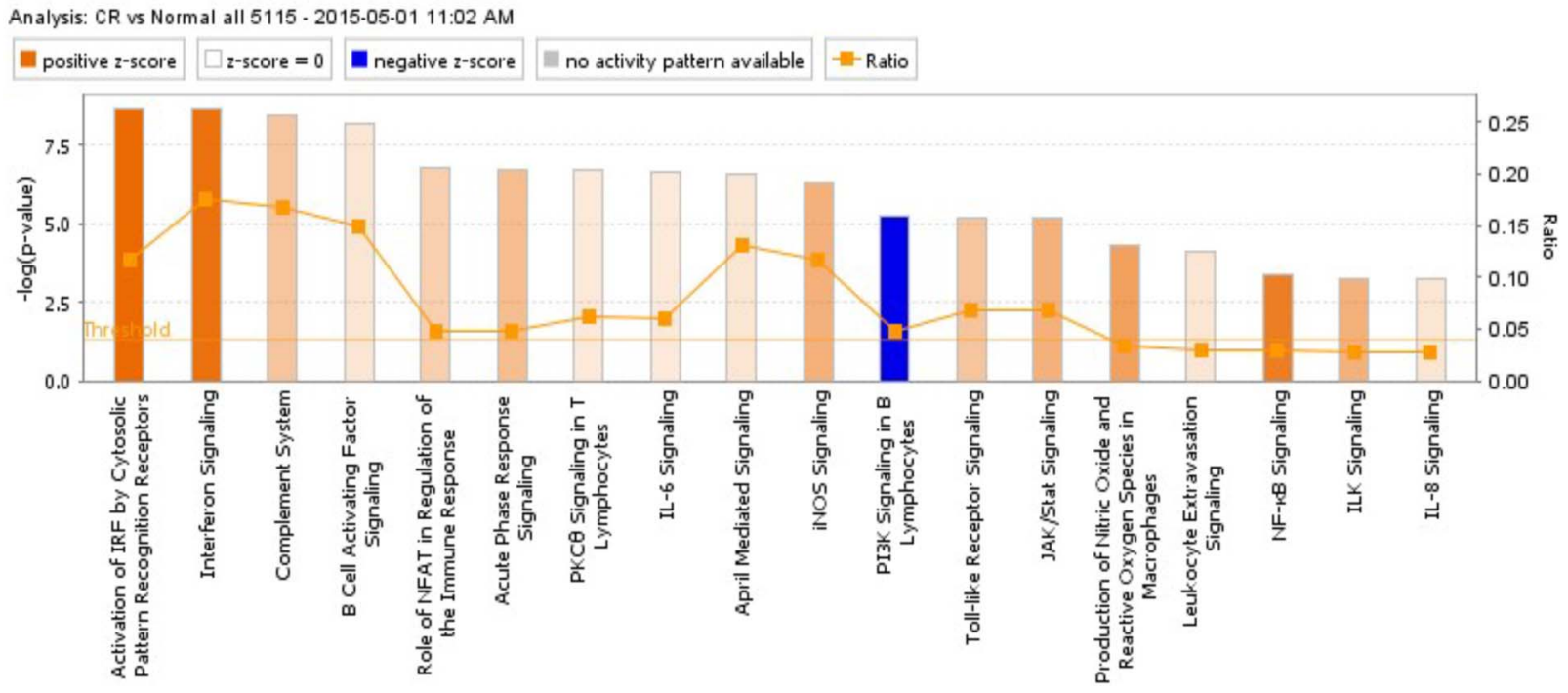

(-) 2000-2015 QIAGEN. All rights resenved.

\section{B Top activated/suppressed immune pathways in NR flares}

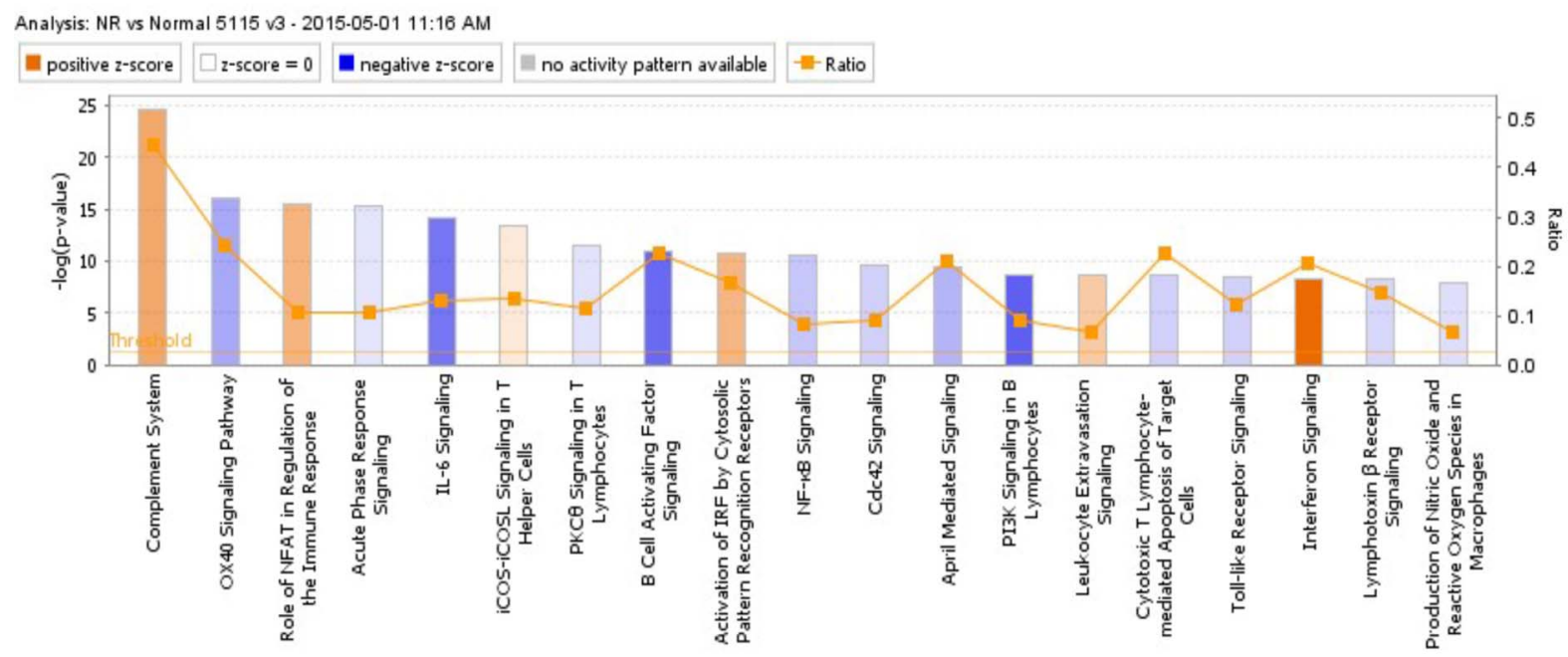

(2000.2015 QIAGEN. All rights reserved.

Figure 3 Canonical immune pathway expression in complete responders (CR) and non-responders (NRs) compared with normal controls at flare. Using Ingenuity Pathway Analysis (IPA), the lupus nephritis (LN) flare groups were compared with normal kidney based on differentially expressed genes. Only canonical pathways where activation status could be determined by IPA were included. The bars reflect the $p$ value for each pathway. The $p$ value measures the likelihood that association between the differentially expressed genes in the data set and the pathway is due to random chance. The smaller the $p$ value, the taller the bar in the figure, and the less likely the association is due to random chance. All the pathways represented had $p$ values $<0.05$ by right-tailed Fisher's exact test and are considered statistically significant. (A) Pathways that were predicted to be activated or suppressed in CR flares compared with normal controls. (B) Pathways that were predicted to be activated or suppressed in NR flares compared with normal controls.

separation in both CR and NR. Interferon-inducible genes appeared to be most responsible for CR clustering while complement genes seemed most responsible for clustering in NR.

Direct comparison between CR and NR transcript expression at flare yielded five differentially expressed genes. The composition of this panel suggests that the function or activity of several immune pathways may distinguish CR from NR. FADD was suppressed in NR compared with CR. FADD binds Fas receptor after it is engaged by fas-ligand (FasL) and activates an apoptosis cascade through procaspases 8 and 10. Fas/FasL has previously been shown to be important for maintaining immune tolerance through elimination of autoreactive 
lymphocytes. $^{42}$ In murine models, Fas/FasL eradicate autoreactive $\mathrm{T}$ and $\mathrm{B}$ cells from germinal centres of secondary lymphoid organs and deficiency in Fas or FasL leads to lupus-like disease. ${ }^{43}{ }^{44}$ Decreased FADD expression in NR may contribute to blunted tolerance and inefficient clearing of apoptotic debris compared with CR flares.

CD274/PD-L1 is a transmembrane protein that interacts with the PD-1 receptor and regulates T cell co-stimulation. It was suppressed in NR relative to CR. The PD-1:PD-L1 pathway is known to eliminate autoreactive $\mathrm{T}$ cells and protect against autoimmunity. ${ }^{45} \mathrm{PD}-1^{-/-}$mice develop lupus-like disease including glomerulonephritis. ${ }^{46}$ Additionally, CD274/PD-L1 has been shown to play an important role in $\mathrm{T}$ regulatory cell generation from naive CD4 T cells. ${ }^{47}$ In human SLE, CD274/PD-L1 is suppressed in flare but returns when disease is in remission. ${ }^{29}$ This suggests that suppression of CD274/PD-L1 as seen in NR may lead to unregulated autoreactive $\mathrm{T}$ cell activation and defective tolerance compared with CR.

Complement is involved in all LN flares; however, the increased expression of C1 components suggests the classical pathway may be more active in NR flares. Finally, increased expression of ITGB2, the integrin betachain subunit for LFA-1, in NR compared with CR suggests that DNA hypomethylation and unregulated $\mathrm{T}$ cell activity may be more prominent in LN flares that end in NR. ${ }^{48}$ LFA-1 overexpression is associated with $\mathrm{T}$ cell DNA hypomethylation and has previously been implicated in promoting autoreactivity and lupus-like disease in experimental animals. ${ }^{49}$

In a second approach to distinguish future CR from NR using the initial diagnostic biopsy, transcript profiles from each group were analysed to identify differentially activated pathways of immune-mediated kidney injury. CR biopsies at flare were characterised by activation of pro-inflammatory (IL-6, NF- $\kappa \mathrm{B}$, TLR signalling) and B cell (BAFF and APRIL) pathways. These data are consistent with the presumed pathogenesis of SLE and LN. In contrast, and unexpectedly, while interferon and complement were upregulated in NR, the same pro-inflammatory and B cell pathways activated in CR were suppressed in NR kidneys.

There are several potential reasons for the molecular differences between CR and NR kidneys at flare. While immunosuppressive medications at the time of biopsy could be confounders, the NR and CR patients were on similar types and levels of therapy at flare, so this seems unlikely. The simplest explanation is that more CR patients were new onset LN compared with NR, which was mainly relapsing LN. Although not statistically significant, NR had more chronic kidney damage than CR, as reflected by the higher average value of the chronicity index. While it is not clear how chronic parenchymal injury may modify the acute inflammatory processes of $\mathrm{LN}$, relapsing $\mathrm{LN}$ may involve different injury pathways than de novo LN and may require a different approach to treatment.
Additionally, differences between CR and NR may be due to the intrinsic molecular heterogeneity of LN. That is, some LN may be driven more by autoreactive B cells and NF-кB-dependent cytokines, while LN in other patients may be more $\mathrm{T}$ cell dependent. ${ }^{36}$ Finally, the timing of the biopsy relative to an individual's point in the LN flare cycle may contribute to differences between CR and NR. Despite performing kidney biopsies on patients with similar clinical findings, it is generally not known when LN begins in any individual patient with SLE. Furthermore, the progression of parenchymal injury and the kidney's response to injury at the molecular level is almost certainly variable between individuals. Thus, patients who had a rapid clinical response to standard-of-care therapy may have been biopsied earlier in their $\mathrm{LN}$ flare than patients who did not respond quickly, and at a time when $\mathrm{NF}-\kappa \mathrm{B}$ and $\mathrm{B}$ cells were highly active. NRs may have evolved to have increased $\mathrm{T}$ cell-dependent injury due to a continued inability to remove autoreactive $\mathrm{T}$ cells as suggested by suppressed FADD and CD274/PD-L1 expression and increased LFA-1 at biopsy.

It is likely that the duration of LN, its molecular heterogeneity and the timing of biopsy and treatment all contribute to the discordant gene expression patterns in CR and NR. No matter what the explanation, these findings suggest that the pathways active at the time of biopsy may influence response to conventional therapy. Additionally, understanding the active molecular pathways in the kidney when initial treatment is being decided could identify patients who would benefit from novel therapies, such as anti-B cell drugs or drugs that restore immune tolerance.

This study has limitations. The sample size is small with four patients in the NR group and five patients in the CR group. The LN cohort was Hispanic and from Argentina, and the controls were from Ohio. It is possible that some of the molecular heterogeneity of the kidney in LN is influenced by race/ethnicity. Therefore, these results may not be generally applicable to all LN patients. Additionally, whole kidney cortex was studied. Because cortex is mostly tubulointerstitum, these data mainly reflect events occurring in the interstitial compartment. There are likely important differences between the glomeruli and tubulointerstitium. Finally, because transcript expression does not necessarily correlate with protein expression, proteomic evaluation of clinical kidney biopsies could compliment transcript analyses, especially when trying to identify new therapeutic targets.

Nonetheless, our data are consistent with and extend previous studies demonstrating the molecular heterogeneity of LN. ${ }^{36} 50-52$ For example, in a study comparing gene expression profiles of murine and human LN, complement, dendritic cell activation, CTLA4 signalling and antigen presentation pathways were differentially expressed at flare, ${ }^{50}$ similar to the pathways we found to be differentially expressed compared with controls. 
Additionally, another investigation using microarray analysis of murine kidneys at different disease stages showed a significant increase in inflammatory gene expression at the onset of proteinuria that improved with treatment and returned to baseline levels at clinical remission. ${ }^{51}$ In a recent repeat biopsy study of patients with active $\mathrm{LN}$, serum markers of inflammation increased at flare and decreased after treatment when remission was achieved. However, a poor histological response correlated with higher IL-17 levels at flare and persistently elevated IL-23 levels after treatment, suggesting that the IL-17/ IL-23 axis may be an important marker of response. ${ }^{53}$

In summary, these data support the use of molecular pathology to analyse LN biopsies at the time of flare diagnosis. Such analyses could identify patients less likely to respond to standard-of-care therapy, who may do better with a novel drug, and what the novel drug should target. The addition of a molecular evaluation to routine histology would facilitate the personalisation of LN treatment and would be expected to improve shortterm response rates and decrease long-term chronic kidney disease. As shown here, this approach is feasible using routinely collected FFPE biopsies. The differentially activated genes and pathways described here will need to be verified in a larger population of patients of different races and ethnicities.

Contributors SVP and AM are first authors on the paper and contributed equally to the specimen collection, data analysis and writing of this manuscript. JV and HS were responsible for the RNA extraction from the biopsy tissue. VA is a clinical pathologist and provided the biospecimens. BL along with AM provided clinical data. JZ and LY are the biostatisticians and conducted the statistical analysis for this work. BHR oversaw the entire project, developed the study and contributed significantly to the writing of the manuscript.

Funding National Institute of Diabetes and Digestive and Kidney Diseases (U01: DK096927); Mallinckrodt Pharmaceuticals (Fellowship Grant: 00033990).

Competing interests None declared

Ethics approval Hospital Fernandez Ethics Board and The Ohio State University Institutional Review Board.

Provenance and peer review Not commissioned; externally peer reviewed.

Data sharing statement No additional data are available.

Open Access This is an Open Access article distributed in accordance with the terms of the Creative Commons Attribution (CC BY 4.0) license, which permits others to distribute, remix, adapt and build upon this work, for commercial use, provided the original work is properly cited. See: http:// creativecommons.org/licenses/by/4.0/

\section{REFERENCES}

1. Iversen P, Brun C. Aspiration biopsy of the kidney. Am J Med 1951;11:324-30.

2. Weening JJ, D'Agati VD, Schwartz MM, et al. The classification of glomerulonephritis in systemic lupus erythematosus revisited. $J \mathrm{Am}$ Soc Nephrol 2004; 15:241-50.

3. Markowitz GS, D'Agati VD. The ISN/RPS 2003 classification of lupus nephritis: an assessment at 3 years. Kidney Int 2007;71:491-5

4. Austin HA III, Muenz LR, Joyce KM, et al. Prognostic factors in lupus nephritis. Contribution of renal histologic data. $A m \mathrm{~J} \mathrm{Med}$ 1983;75:382-91.
5. Morel-Maroger L, Mery JP, Droz D, et al. The course of lupus nephritis: contribution of serial renal biopsies. Adv Nephrol Necker Hosp 1976;6:79-118.

6. Alsuwaida A, Husain S, Alghonaim M, et al. Strategy for second kidney biopsy in patients with lupus nephritis. Nephrol Dial Transplant 2012;27:1472-8.

7. Esdaile JM, Joseph L, MacKenzie T, et al. The pathogenesis and prognosis of lupus nephritis: information from repeat renal biopsy. Semin Arthritis Rheum 1993;23:135-48.

8. Gunnarsson I, Sundelin B, Heimburger M, et al. Repeated renal biopsy in proliferative lupus nephritis--predictive role of serum C1q and albuminuria. J Rheumatol 2002;29:693-9.

9. Moroni G, Pasquali S, Quaglini S, et al. Clinical and prognostic value of serial renal biopsies in lupus nephritis. Am J Kidney Dis 1999;34:530-9.

10. Hahn BH, McMahon MA, Wilkinson A, et al. American College of Rheumatology. American College of Rheumatology guidelines for screening, treatment, and management of lupus nephritis. Arthritis Care Res (Hoboken) 2012;64:797-808.

11. Golubeva Y, Salcedo R, Mueller C, et al. Laser capture microdissection for protein and NanoString RNA analysis. Methods Mol Biol 2013;931:213-57.

12. Vaes E, Khan M, Mombaerts P. Statistical analysis of differential gene expression relative to a fold change threshold on NanoString data of mouse odorant receptor genes. BMC Bioinformatics 2014:15:39.

13. Geiss GK, Bumgarner RE, Birditt B, et al. Direct multiplexed measurement of gene expression with color-coded probe pairs. Nat Biotechnol 2008;26:317-25.

14. Reis PP, Waldron L, Goswami RS, et al. mRNA transcript quantification in archival samples using multiplexed, color-coded probes. BMC Biotechnol 2011;11:46.

15. Tam S, de Borja R, Tsao MS, et al. Robust global microRNA expression profiling using next-generation sequencing technologies. Lab Invest 2014;94:350-8.

16. Sartor MA, Tomlinson CR, Wesselkamper SC, et al. Intensity-based hierarchical Bayes method improves testing for differentially expressed genes in microarray experiments. BMC Bioinformatics 2006;7:538.

17. Johnson R, Wichem DW. Applied Multivariate Statistical Analysis. New Jersey: Prentice Hall, 1998

18. Arima $\mathrm{K}$, Kinoshita A, Mishima $\mathrm{H}$, et al. Proteasome assembly defect due to a proteasome subunit beta type 8 (PSMB8) mutation causes the autoinflammatory disorder, Nakajo-Nishimura syndrome. Proc Natl Acad Sci USA 2011:108:14914-19.

19. Lan HY. Diverse roles of TGF-beta/Smads in renal fibrosis and inflammation. Int J Biol Sci 2011;7:1056-67.

20. Okuda S, Languino LR, Ruoslahti E, et al. Elevated expression of transforming growth factor-beta and proteoglycan production in experimental glomerulonephritis. Possible role in expansion of the mesangial extracellular matrix. J Clin Invest 1990;86:453-62.

21. Yamamoto K, Loskutoff DJ. Expression of transforming growth factor-beta and tumor necrosis factor-alpha in the plasma and tissues of mice with lupus nephritis. Lab Invest 2000;80:1561-70.

22. Ruffell D, Mourkioti F, Gambardella A, et al. A CREB-C/EBP beta cascade induces M2 macrophage-specific gene expression and promotes muscle injury repair. Proc Natl Acad Sci USA 2009;106:17475-80.

23. Kaleta B. Role of osteopontin in systemic lupus erythematosus. Arch Immunol Ther Exp (Warsz) 2014;62:475-82.

24. Miyazaki T, Ono M, Qu WM, et al. Implication of allelic polymorphism of osteopontin in the development of lupus nephritis in MRL/lpr mice. Eur J Immunol 2005;35:1510-20.

25. Rullo OJ, Woo JM, Parsa MF, et al. Plasma levels of osteopontin identify patients at risk for organ damage in systemic lupus erythematosus. Arthritis Res Ther 2013;15:R18.

26. Molineros JE, Maiti AK, Sun C, et al. Admixture mapping in lupus identifies multiple functional variants within IFIH1 associated with apoptosis, inflammation, and autoantibody production. PLoS Genet 2013;9:e1003222.

27. Mehta J, Genin A, Brunner M, et al. Prolonged expression of CD154 on CD4 T cells from pediatric lupus patients correlates with increased CD154 transcription, increased nuclear factor of activated T cell activity, and glomerulonephritis. Arthritis Rheum 2010;62:2499-509.

28. Akhabir L, Sandford A. Genetics of interleukin 1 receptor-like 1 in immune and inflammatory diseases. Curr Genomics 2010;11:591-606.

29. Ou JN, Wiedeman AE, Stevens AM. TNF-alpha and TGF-beta counter-regulate PD-L1 expression on monocytes in systemic lupus erythematosus. Sci Rep 2012;2:295. 
30. Eyre S, Hinks A, Bowes J, et al. Overlapping genetic susceptibility variants between three autoimmune disorders: rheumatoid arthritis, type 1 diabetes and coeliac disease. Arthritis Res Ther 2010;12:R175.

31. Baechler EC, Batliwalla FM, Karypis G, et al. Interferon-inducible gene expression signature in peripheral blood cells of patients with severe lupus. Proc Natl Acad Sci USA 2003;100:2610-15.

32. Bao L, Quigg RJ. Complement in lupus nephritis: the good, the bad, and the unknown. Semin Nephrol 2007;27:69-80.

33. Birmingham DJ, Irshaid F, Nagaraja HN, et al. The complex nature of serum $\mathrm{C} 3$ and $\mathrm{C} 4$ as biomarkers of lupus renal flare. Lupus 2010;19:1272-80.

34. Dall'era MC, Cardarelli PM, Preston BT, et al. Type I interferon correlates with serological and clinical manifestations of SLE. Ann Rheum Dis 2005;64:1692-7.

35. Karonitsch T, Feierl E, Steiner CW, et al. Activation of the interferongamma signaling pathway in systemic lupus erythematosus peripheral blood mononuclear cells. Arthritis Rheum 2009;60:1463-71.

36. Peterson KS, Huang JF, Zhu J, et al. Characterization of heterogeneity in the molecular pathogenesis of lupus nephritis from transcriptional profiles of laser-captured glomeruli. J Clin Invest 2004:113:1722-33.

37. Rönnblom L, Pascual V. The innate immune system in SLE: type I interferons and dendritic cells. Lupus 2008;17:394-9.

38. Yasuda K, Richez C, Maciaszek JW, et al. Murine dendritic cell type I IFN production induced by human IgG-RNA immune complexes is IFN regulatory factor (IRF)5 and IRF7 dependent and is required for IL-6 production. J Immunol 2007;178:6876-85.

39. Hsieh C, Chang A, Brandt D, et al. Predicting outcomes of lupus nephritis with tubulointerstitial inflammation and scarring. Arthritis Care Res (Hoboken) 2011;63:865-74.

40. Kavai M, Szegedi G. Immune complex clearance by monocytes and macrophages in systemic lupus erythematosus. Autoimmun Rev 2007;6:497-502.

41. Kinloch AJ, Chang A, Ko K, et al. Vimentin is a dominant target of in situ humoral immunity in human lupus tubulointerstitial nephritis. Autoimmun Rev 2014;66:3359-70.
42. McNally J, Yoo DH, Drappa J, et al. Fas ligand expression and function in systemic lupus erythematosus. $J$ Immunol 1997;159:4628-36.

43. Nagata S, Golstein P. The Fas death factor. Science 1995:267:1449-56.

44. Rathmell JC, Cooke MP, Ho WY, et al. CD95 (Fas)-dependent elimination of self-reactive $B$ cells upon interaction with CD4+ T cells. Nature 1995;376:181-4.

45. Francisco LM, Sage PT, Sharpe AH. The PD-1 pathway in tolerance and autoimmunity. Immunol Rev 2010;236:219-42.

46. Nishimura $\mathrm{H}$, Nose M, Hiai $\mathrm{H}$, et al. Development of lupus-like autoimmune diseases by disruption of the PD-1 gene encoding an ITIM motif-carrying immunoreceptor. Immunity 1999;11: $141-51$

47. Francisco LM, Salinas VH, Brown KE, et al. PD-L1 regulates the development, maintenance, and function of induced regulatory T cells. J Exp Med 2009;206:3015-29.

48. Smith $\mathrm{A}$, Stanley $\mathrm{P}$, Jones $\mathrm{K}$, et al. The role of the integrin LFA-1 in T-lymphocyte migration. Immunol Rev 2007;218:135-46.

49. Lu Q, Kaplan M, Ray D, et al. Demethylation of ITGAL (CD11a) regulatory sequences in systemic lupus erythematosus. Arthritis Rheum 2002;46:1282-91.

50. Berthier CC, Bethunaickan R, Gonzalez-Rivera T, et al. Cross-species transcriptional network analysis defines shared inflammatory responses in murine and human lupus nephritis. $J$ Immunol 2012;189:988-1001.

51. Bethunaickan R, Berthier CC, Zhang W, et al. Identification of stage specific genes associated with lupus nephritis and response to remission induction in (NZBXNZW)F1 and NZM2410 mice. Arthritis Rheum 2014;66:2246-58.

52. Teramoto K, Negoro N, Kitamoto K, et al. Microarray analysis of glomerular gene expression in murine lupus nephritis. J Pharmacol Sci 2008;106:56-67.

53. Zickert A, Amoudruz P, Sundström Y, et al. IL-17 and IL-23 in lupus nephritis-association to histopathology and response to treatment. BMC Immunol 2015;16:7. 